Fertil Steril. 2014 July ; 102(1): 178-182. doi:10.1016/j.fertnstert.2014.03.017.

\title{
Peri-implantation Intercourse Lowers Fecundability
}

\author{
Anne Z. Steiner, MD, MPH ${ }^{a}$, David A. Pritchard, MS ${ }^{b}$, Steven L. Young, MD, PhD ${ }^{a}$, and Amy \\ H. Herring, $S_{c}{ }^{c}$ \\ aDepartment of Obstetrics and Gynecology, University of North Carolina, Chapel Hill, NC \\ bepartment of Biostatistics, University of North Carolina, Chapel Hill, NC \\ ${ }^{c}$ Carolina Population Center, University of North Carolina, Chapel Hill, NC
}

\begin{abstract}
Objective-To determine the impact of sexual intercourse around the time of implantation on the probability of achieving a pregnancy.
\end{abstract}

Design-Time-to-pregnancy cohort using day-specific probability of pregnancy modeling to account for intercourse during the fertile window.

Setting-Community cohort.

Patient(s) -Women trying to conceive naturally, ages 30-44 without known infertility.

Intervention(s)-None.

Main Outcome Measure(s)-Positive pregnancy test

Result(s)-A total of 564 women provided 1,332 complete cycles for analysis. Intercourse frequency during the fertile window and during the peri-implantation window was significantly correlated. Cycles in which couples had 2 or more days with intercourse during the implantation window were significantly less likely to result in a positive pregnancy test compared to cycles in which couples didn't have intercourse in this window, after adjusting for age, race, history of regular menstrual cycles, previous pregnancy, and body mass index (Fecundability Ratio $=0.62$, 95\% Confidence Interval: 0.42-0.91).

Conclusions-Intercourse during the peri-implantation window may be detrimental to natural fertility. Methods that allow couples to time intercourse to the fertile window may decrease time to pregnancy by not only increasing the probability of fertilization but also decreasing the probability of failed implantation.

(C) 2014 American Society for Reproductive Medicine. Published by Elsevier Inc. All rights reserved.

Corresponding Author: Anne Z. Steiner, MD, MPH, Associate Professor, Department of Obstetrics and Gynecology, UNC School of Medicine, CB \#7570, Old Clinic Building, Chapel Hill, NC 27599, (919) 966-5283, (919) 966-5214, asteiner@med.unc.edu.

Publisher's Disclaimer: This is a PDF file of an unedited manuscript that has been accepted for publication. As a service to our customers we are providing this early version of the manuscript. The manuscript will undergo copyediting, typesetting, and review of the resulting proof before it is published in its final citable form. Please note that during the production process errors may be discovered which could affect the content, and all legal disclaimers that apply to the journal pertain.

DISCLOSURES: None. 


\section{Keywords}

Intercourse; fecundability; implantation; time-to-pregnancy

\section{Introduction}

Previously, Wilcox et al. showed that the fertile window includes the six days prior to and including the day of ovulation (1). For natural conception to occur, procreative intercourse must occur during this window. Theoretically, couples, who are having intercourse at least twice a week, should succeed in having intercourse during this interval. However, previous studies have shown that methods, which allow a couple to time intercourse to the fertile window, lead to higher pregnancy rates $(2,3)$. One possible explanation for this finding is that intercourse during both the fertile and non-fertile window lowers pregnancy rates.

Hypothetically, intercourse around the time of implantation, could result in uterine contractions, disrupting the implantation process, displacing the implanted embryo, or expelling the embryo from the uterus. Previous studies have shown increased myometrial activity during intercourse; myometrial activity increases with female orgasm (4). Uterine contractions around the time of embryo transfer during assisted reproductive technology lowers pregnancy rates $(5,6)$. Medications used to inhibit myometrial contractions around the time of embryo transfer have been shown to improve implantation rates (7). Based on these theories, some clinicians recommend that couples abstain from intercourse following embryo transfer during assisted reproductive technology cycles.

Seminal fluid also contains many potent factors that modify and directly elicit a complex maternal immune response (8). Seminal factors include IL8, CXCL12, CCL2, soluble HLAG, TGF-beta, and PGE series prostaglandins, as well as specific antigens presented on the sperm cells (8). The introduction of semen rapidly induces a strong inflammatory response by the female reproductive tract, as shown in multiple species, whose superficial cervical and endometrial tissues undergo large changes in leukocyte population lasting about two days (9-11). Although human data are limited, they support very similar actions of seminal fluid to those seen in animal models (12). After the initial inflammatory response to semen, a secondary tolerogenic response occurs that is likely protective for pregnancy (8). Thus, sexual intercourse at the time of embryo implantation could theoretically induce an unwanted pro-inflammatory response which could interfere with normal embryo implantation.

We sought to determine if intercourse around the time of implantation affected fecundability, the probability of achieving a positive pregnancy test in a given cycle. We hypothesized that intercourse during the peri-implantation window, including implantation and the first couple of days following implantation, would lower the probability of conceiving in a given cycle. 


\section{Materials and Methods}

This study was a secondary analysis of data obtained from Time to Conceive, (TTC) a prospective time-to-pregnancy study, designed to determine the ability of biomarkers of ovarian aging to predict reproductive potential $(13,14)$. IRB approval was obtained for this research. Women were recruited to TTC from the community using flyers, mass email, informational letters, internet, radio, and television advertising, print ads, and community blogs. All advertising materials directed interested women to an informational website or study telephone number. Women were screened for eligibility using a telephone interview. Women, who were eligible for TTC, were between the ages of 30 and 44 years of age, had been attempting to conceive naturally for 3 or fewer months, and did not have risk factors for infertility (e.g. history of polycystic ovarian syndrome, endometriosis, or pelvic inflammatory disease). After consent was obtained, participants complete a self-reported, online baseline survey of demographics, height, weight, and medical history-for both herself and her male partner-as well as behaviors including tobacco, alcohol and caffeine use. While attempting conceive, women completed on online daily diary for up to 4 months or up to the time of pregnancy detection, in which they recorded vaginal bleeding, intercourse, results of ovulation prediction testing (ovulation predictor kit or cervical mucus monitoring), and results of pregnancy tests. Women were not required to monitor for ovulation, but were asked to record the results if they did check their cervical mucus and/or ovulation predictor kit. Participants were provided free home pregnancy tests (sensitivity=20 milli-international units human chorionic gonadotropin/ml) and standardized pregnancy test instructions. Initially women were instructed to test with missed menses. Later the protocol was modified to instruct women to test on cycle days $28,31,34$, etc. until a positive test was noted or menses began.

Data from the baseline survey and daily diary were used to construct menstrual cycles. The start of a menstrual cycle was defined by 1) at least 2 consecutive days of bleeding (not spotting), 2) three or more days of bleeding and spotting occurring at least 15 days from the start of the last cycle, or 3) participant-reported last menstrual period (if entire cycle not observed). For each menstrual cycle, day of ovulation was estimated using the calendar method (15). Ovulation was assumed to have occurred 14 days prior to the first day of menses or first positive home pregnancy test, with the fertile window designated as extending from 5 days before to 3 days after the estimated day of ovulation, as defined above $(16,17)$. The peri-implantation window was defined as extending 5 days after ovulation to 9 days after ovulation. Using the calendar method this corresponds to the 3 to 9 days prior to the end of the menstrual cycle (whether ending in menses or a positive pregnancy test). For this analysis, cycles were not included in the analysis if the entire fertile window and peri-implantation window were not observed.

The number of days with intercourse during the peri-implantation window was determined for each cycle by responses in the daily diary. For analyses, number of days with intercourse during the peri-implantation window was categorized as none, one, or two or more. Pregnancy, the outcome for this study, was defined by the first report of a positive home pregnancy test. 
Pearson correlations, Kruskal-Wallis tests, and chi-square tests were used to compare demographics and potential covariates (subject age, race, education level, previous pregnancy, BMI, ovulation predictor kit use, smoking, hormonal contraception in the preceding year, partner age, partner race, and partner education) between categories of periimplantation intercourse. For the models, we included those covariates which were strongly associated with fecundability in our study or which had been identified in multiple prior studies as related to fecundability, even if these variables were not statistically significant in our study. Potential covariates that were highly correlated with other predictors thought to have a greater relevance (i.e. partner age is highly correlated with subject age) were also excluded from the primary analysis.

The day-specific probabilities model by Scarpa and Dunson (18) (and used in prior analyses) $(13,14)$ was used. Intercourse patterns are accommodated through inclusion of indicators of intercourse on each day of the fertile window that restrict the probability of conception on a non-intercourse day to be 0 .This model provides Bayesian estimates of the fecundability ratio (FR) and an accompanying 95\% credible interval (CI). In this model a FR $<1.0$ suggests reduced fecundability. The day-specific probabilities model allows one to determine the effect of peri-implantation intercourse on achieving a pregnancy independent of intercourse during the implantation window via inclusion of indicator variables for number of peri-implantation intercourse days as a covariate. Other covariates used in the model include maternal age (collapsed into 3 categories for modeling: 30-34, 35-37, and 38-44), race (non-Hispanic white or non-white), history of previous pregnancy (dichotomized as any/none), maternal BMI, and self-reported history of regular menstrual cycles (yes/no). To address the need for left truncation, attempt cycle at enrollment was added to the model as a covariate; however, addition of the covariate did not affect the estimates or confidence intervals, thus it was not retained in the final model.

\section{Results}

A total of 743 women were enrolled in the study between April 2008 and August 2012. Seventy-six percent $(\mathrm{N}=564)$ of women completed the baseline survey and at least one cycle of daily diaries and were therefore included in this analysis. There were no significant differences in covariates examined in this study between the women who completed the daily diaries and those that did not. Of the analysis cohort, $70 \%$ of women were $<35$ years of age, $18 \%$ were $35-37$, and $12 \%$ were 38 years or older. Participants tended to be White (77\%), highly educated (62\% with a graduate degree), previously pregnant $(53 \%)$ and of normal BMI (82\%). Fifteen percent of participants were obese (BMI 230 ) and $3 \%$ were underweight $(\mathrm{BMI}<18.5)$.

A total of 1332 cycles were included in the analysis. Twenty percent of cycles resulted in a pregnancy (cohort fecundability). $46 \%$ of couples had 2-3 days of intercourse during the fertile window. During the peri-implantation window (7 days duration) of a cycle, $32 \%$ of couples had no intercourse, $30 \%$ had intercourse on 1 day, and $19 \%$ had intercourse on 2 day, and $19 \%$ on 3 or more days. The probability of intercourse on any day in the periimplantation window was $14-16 \%$. Number of days with intercourse during the fertile 
window was highly correlated with number of days of intercourse during the periimplantation window $(\mathrm{r}=0.48, \mathrm{p}<0.001)$.

During the first cycle in the study, 28\% (N=159) of women did not have intercourse during the peri-implantation window, $30 \%(\mathrm{~N}=166)$ had intercourse on 1 day, and $42 \%(\mathrm{~N}=239)$ had intercourse on 2 or more days. Women who had intercourse on 2 or more days (in the first cycle) tended not to be White. There were no differences in regards to age, education, body mass index, ovulation predictor kit use, and smoking status (Table 1) by number of days of intercourse during the peri-implantation window.

The unadjusted and adjusted fecundability ratios for intercourse during the peri-implantation window as determined using day-specific probabilities models, which adjust for intercourse during the fertile window, were calculated. As number of days with intercourse during the peri-implantation window increased, the probability of pregnancy in that cycle appeared to decrease. Couples who had intercourse on 2 or more days during the peri-implantation window were approximately $40 \%$ less likely to conceive compared to couples that did not have intercourse during the peri-implantation window in unadjusted models (FR 0.59, 95\% CI: 0.40-0.86) and after adjusting for age, BMI, race, previous pregnancy, and presence of regular menses ( $\mathrm{FR}=0.62,95 \% \mathrm{CI}$ : 0.42-0.91). With further exploration we noted that the probability of pregnancy decreased as number of days with intercourse during the periimplantation window increased (Table 2).

A sensitivity analysis was conducted including only cycles in which a woman recorded a positive result on her ovulation predictor kit. Ovulation was defined as the day following the first positive LH test. The implantation window was defined as the 6 to 10 days after $\mathrm{LH}$ surge (LH+ 6-10). The fertile window was defined as the 4 days prior to $\mathrm{LH}$ surge to one day after the LH surge (LH-4 to +1). A total of 156 women with 226 cycles contributed to this analysis. In this subgroup, women who had intercourse during the implantation window were $26 \%$ less likely to conceive compared to women that did not have intercourse during the implantation window (FR 0.74, 95\% CI: 0.38-1.28), though this result did not reach statistical significance.

\section{Discussion}

This prospective study sought to determine the impact of intercourse around the time of implantation on achieving a positive pregnancy test given intercourse during the preceding fertile window. In this study couples that had more frequent intercourse during the fertile window tended to have more intercourse during the peri-implantation window. White women tended to have less frequent intercourse during the peri-implantation window. Age, education, BMI, ovulation predicator kit use, and smoking status did not differ by frequency of intercourse during the peri-implantation window. Two or more days with intercourse during the peri-implantation window was associated with a significantly lower probability of pregnancy given prior intercourse during the fertile window.

Couples, who tended to have frequent intercourse during the fertile window tended to be more sexually active during the peri-implantation window. Therefore, if one were to simply 
examine the relationship between peri-implantation intercourse and fecundability without adjusting for intercourse patterns during the fertile window, one might falsely conclude that peri-implantation intercourse increases fecundability. However, it is the frequency of intercourse during the fertile window, a six day window including the 5 days prior and day of ovulation, which increases probability of pregnancy. One of the strengths of this study is the use of daily dairies and day-specific probability modeling, which allow one to adjust for intercourse during the fertile window to determine the independent effect of periimplantation intercourse on fecundability.

As intercourse frequency during the peri-implantation window increased, fecundability decreased. Couples that had 2 or more acts of intercourse during the peri-implantation window were significantly less likely to get pregnant compared to couples that did not have intercourse during this window. Previous studies of the impact of non-procreative intercourse on fertility have 1) examined intercourse during assisted reproductive technology and 2) largely focused on intercourse around the time of oocyte retrieval. Marconi et al. found that couples who were undergoing gamete intrafallopian tube transfer were more likely to conceive if they had intercourse around the time of hCG administration (20). They did not examine the impact of peri-implantation intercourse on outcomes. Similarly, Bellinge et al. found that vaginal insemination at the time of oocyte retrieval during in vitro fertilization increased pregnancy rates (21). An increase in pregnancy rates was observed among women with tubal obstruction, suggesting that the increase was not due to in vivo fertilization. However, a second study of slightly large size failed to show a benefit (22).

Only one study has examined the impact of intercourse remote from egg retrieval. Tremellen et al. examined the impact of intercourse on the evening prior to egg retrieval and the evening following a day 2 embryo transfer (23). They did not find a significant increase in pregnancy rates in the group that had intercourse and the group that didn't; however, implantation rate was higher in the intercourse group. It is possible that the intercourse episodes had contradictory effects (intercourse prior increasing pregnancy rates and intercourse following embryo transfer decreasing rates). In the same study they examined the impact of a single episode of intercourse around the time of a day 3 frozen embryo transfer. Pregnancy rates did not significantly differ between those groups that had intercourse at time of transfer and those that didn't. While these results appear to differ from ours, the timing and frequency of intercourse differed between our studies. Tremellen et al. examined the impact of a single episode prior to implantation. This would suggest that multiple episodes during implantation (but not prior) negatively affect implantation. It is still possible that semen exposure prior to implantation leads to immune modulation important for successful implantation (8) and that high levels of semen exposure during the periimplantation phase can cause pro-inflammatory changes that overcome the tolerogenic effect of prior peri-ovulatory exposure. Another important possibility is that semen components interrupt the complex maternal-embryonic cross signaling that is thought to occur during implantation.

This study does have limitations. The peri-implantation window was defined by the calendar method. Thus we may not have truly isolated the implantation window. However, we would 
have thought that this would have biased the findings toward the intercourse group. Three women ( $1 \%$ of pregnancies) conceived outside the fertile window identified by the calendar method and were not included in the analysis. Alternatively women may have more intercourse during anovulatory cycles. Such a relationship would bias our findings against the intercourse group. We did conduct a sensitivity analysis, which only included women who used ovulation predictor kits. Effect estimates for our sensitivity analysis were consistent with our full cohort estimate. An ideal study would include a biomarker for ovulation, daily diaries, and would mask participants to biomarker results.

We did not use progesterone levels or human chorionic gonadotropin (HCG) levels to determine the implantation window. Progesterone levels fluctuate greatly within a day and over the luteal phase within and between women. Maternal HCG levels can only be measured once all the steps of implantation have occurred (apposition, attachment, and invasion). HCG levels are also dependent on embryo viability. Diaries did not include information on orgasm or condom use. Therefore we can not comment as to whether the lower pregnancy rates were due to semen exposure, the act of intercourse, or orgasm. As seen with most prospective, time-to pregnancy cohorts, the cohort contained a high proportion of white, older, highly educated women. These findings may not be generalizable to other groups. The strengths of this study include the size, modeling to accommodate for intercourse during the fertile window, and adjustment for potential confounders.

In conclusion, intercourse during the peri-implantation window may be detrimental to natural fertility. However, we do not recommend a change in practice patterns based on this single study. The results of this study need to be confirmed in other time-to-pregnancy cohorts and in studies of women undergoing assisted reproductive technology. Methods that allow couples to time intercourse to the fertile window may decrease time to pregnancy not only by increasing the probability of fertilization but also by decreasing the probability of failed implantation.

\section{Acknowledgments}

SUPPORT: NICHD R21 HD060229, NICHD R01 HD067683, NIEHS T32ES007018.

\section{References}

1. Wilcox AJ, Weinberg CR, Baird DD. Timing of sexual intercourse in relation to ovulation: effects on the probability of conception, survival of the pregnancy, and sex of the baby. NEJM. 1995; 333:1517-1521. [PubMed: 7477165]

2. Evans-Hoeker E, Pritchard DA, Long DL, Herring AH, Stanford JB, Steiner AZ. Cervical mucus monitoring prevalence and associated fecundability in women trying to conceive. Fertil Steril. 2013; 100:1033-1038. [PubMed: 23850303]

3. Robinson JE, Wakelin M, Ellis JE. Increased pregnancy rate with use of the Clearblue Easy Fertility Monitor. Fertil Steril. 2007; 87:329-334. [PubMed: 17074329]

4. Fox CA, Wolff HS, Baker JA. Measurement of intra-vaginal and intra-uterine pressures during human coitus by radio-telemetry. J Reprod Fertil. 1970; 22:243-251. [PubMed: 5428946]

5. Fanchin R, Righini C, Olivennes F, Taylor S, de Ziegler D, Frydman R. Uterine contractions at the time of embryo transfer alter pregnancy rates after in-vitro fertilization. Hum Reprod. 1998; 13:1968-1974. [PubMed: 9740459] 
6. Lesny P, Killick SR, Robinson J, Raven G, Maguiness SD. Junctional zone contractions and embryo transfer: is it safe to use a tenaculum? Hum Reprod. 1999; 14:2367-2370. [PubMed: 10469712]

7. Lan VT, Khang VN, Nhu GH, Tuong HM. Atosiban improves implantation and pregnancy rates in patients with repeated implantation failure. Reprod Biomed Online. 2012; 25:254-260. [PubMed: 22818095]

8. Robertson SA, Prins JR, Sharkey DJ, Moldenhauer LM. Seminal Fluid and the Generation of Regulatory T Cells for Embryo Implantation. Am J of Reprod Immunol. 2013; 69:315-330. [PubMed: 23480148]

9. Piazzon I, Matusevich M, Deroche A, Nepomnaschy I, Pasqualini CD. Early increase in graftversus-host reactivity during pregnancy in the mouse. J Reprod Immunol. 1985; 8:129-137. [PubMed: 3879270]

10. De M, Choudhuri R, Wood GW. Determination of the number and distribution of macrophages, lymphocytes, and granulocytes in the mouse uterus from mating through implantation. J Leukoc Biol. 1991; 50:252-262. [PubMed: 1856596]

11. Lovell JW, Getty R. Fate of semen in the uterus of the sow: histologic study of endometrium during the 27 hours after natural service. Am J Vet Res. 1968; 29:609-625. [PubMed: 5688982]

12. Robertson SA, Bromfield JJ, Tremellen KP. Seminal 'priming' for protection from pre-eclampsia-a unifying hypothesis. J Reprod Immunol. 2003; 59:253-265. [PubMed: 12896827]

13. Steiner AZ, Herring AH, Kesner JS, Meadows JW, Stanczyk FZ, Hoberman S, et al. Antimullerian hormone as a predictor of natural fecundability in women aged 30-42 years. Obstet Gynecol. 2011; 117:798-804. [PubMed: 21422850]

14. Steiner AZ, Long DL, Herring AH, Kesner JS, Meadows JW, Baird DD. Urinary folliclestimulating hormone as a measure of natural fertility in a community cohort. Reprod Sci. 2013; 20:549-556. [PubMed: 23171685]

15. Arevalo M, Sinai I, Jennings V. A fixed formula to define the fertile window of the menstrual cycle as the basis of a simple method of natural family planning. Contraception. 1999; 60:357360. [PubMed: 10715371]

16. Evans-Hoeker E, Pritchard DA, Long DL, Herring AH, Stanford JB, Steiner AZ. Cervical mucus monitoring prevalence and associated fecundability in women trying to conceive. Fertil Steril. 2013; 100:1033-1038. [PubMed: 23850303]

17. Steiner AZ, Long DL, Tanner C, Herring AH. Effect of vaginal lubricants on natural fertility. Obstet Gynecol. 2012; 120:44-51. [PubMed: 22914390]

18. Scarpa B, Dunson DB. Bayesian selection of predictors of conception probabilities across the menstrual cycle. Paediatr Perinat Epidemiol. 2006; 20(Suppl 1):30-37. [PubMed: 17061971]

19. Moos MK, Petersen R, Meadows K, Melvin CL, Spitz AM. Pregnant women's perspectives on intendedness of pregnancy. Womens Health Issues. 1997; 7:385-392. [PubMed: 9439199]

20. Marconi G, Auge L, Oses R, Quintana R, Raffo F, Young E. Does sexual intercourse improve pregnancy rates in gamete intrafallopian transfer? Fertil Steril. 1989; 51:357-359. [PubMed: 2643533]

21. Bellinge BS, Copeland CM, Thomas TD, Mazzucchelli RE, O'Neil G, Cohen MJ. The influence of patient insemination on the implantation rate in an in vitro ferilization and embryo transfer program. Fertil Steril. 1986; 46:252-256. [PubMed: 3732531]

22. Fishel S, Webster J, Jackson P, Faratian B. Evaluation of high vaginal insemination at oocyte recovery in patients undergoing in vitro fertilization. Fertil Steril. 1989; 51:135-138. [PubMed: 2910708]

23. Tremellen KP, Valbuena D, Landeras J, Ballesteros A, Martinez J, Mendoza S, et al. The effect of intercourse on pregnancy rates during assisted human reproduction. Hum Reprod. 2000; 15:26532658. [PubMed: 11098040]

24. Kruger THC, Leeners B, Naegeli E, Schmidlin S, Schedlowski M, Hartmann U, et al. Prolactin secretory rhythm in women: immediate and long-term alterations after sexual contact. Hum Reprod. 2011; 27:1139-1143. [PubMed: 22333984] 
Table 1

Baseline characteristics by number of days with intercourse during the peri-implantation window during the first menstrual cycle following enrollment $(\mathrm{N}=564)$. Data are presented as $\mathrm{N}(\%)$.

\begin{tabular}{|c|c|c|c|c|}
\hline & \multicolumn{4}{|c|}{$\begin{array}{l}\text { Number of days with intercourse during the peri- } \\
\text { implantation window }\end{array}$} \\
\hline & $\begin{array}{l}\text { None } \\
(\mathrm{N}=159)\end{array}$ & $\begin{array}{c}\text { One } \\
(\mathrm{N}=166)\end{array}$ & $\begin{array}{l}\text { Two or More } \\
(\mathbf{N}=239)\end{array}$ & P-value ${ }^{a}$ \\
\hline Age (years) & & & & 0.48 \\
\hline$<35$ & $105(66)$ & $120(72)$ & $167(70)$ & \\
\hline $35-37$ & $40(25)$ & $38(23)$ & $51(21)$ & \\
\hline$>38$ & $14(9)$ & $8(5)$ & $21(9)$ & \\
\hline Race & & & & 0.004 \\
\hline Non-Hispanic Caucasian & $132(83)$ & $135(81)$ & $168(70)$ & \\
\hline Other & $27(17)$ & $31(19)$ & $71(30)$ & \\
\hline Education level & & & & 0.63 \\
\hline Less than college degree & $36(23)$ & $35(21)$ & $51(21)$ & \\
\hline College degree & $100(63)$ & $105(63)$ & $144(60)$ & \\
\hline Some graduate/masters & $8(5)$ & $10(6)$ & $24(10)$ & \\
\hline Complete post-graduate & $15(9)$ & $16(10)$ & $20(9)$ & \\
\hline Nulligravid & $72(45)$ & $91(55)$ & $104(44)$ & 0.07 \\
\hline Regular menstrual cycles & $143(88)$ & $150(90)$ & $200(85)$ & 0.35 \\
\hline Body mass index $\left(\mathrm{kg} / \mathrm{m}^{2}\right)$ & & & & 0.96 \\
\hline$<18.5$ & $6(4)$ & $5(3)$ & $6(3)$ & \\
\hline $18.5-29.9$ & $133(82)$ & $138(83)$ & $192(82)$ & \\
\hline 230 & $23(14)$ & $24(14)$ & $37(16)$ & \\
\hline Ovulation Prediction Kit use & $45(28)$ & $37(22)$ & $56(23)$ & 0.40 \\
\hline Current Smoker & $3(2)$ & $1(0.6)$ & $5(2)$ & 0.47 \\
\hline Partner's age (years) & & & & 0.82 \\
\hline$<50$ & $157(99)$ & $164(99)$ & $236(99)$ & \\
\hline 50 or more & $1(1)$ & $2(1)$ & $3(1)$ & \\
\hline Partner race & & & & $<0.001$ \\
\hline Non-Hispanic White & $129(81)$ & $135(81)$ & $165(69)$ & \\
\hline Other & $30(19)$ & $31(19)$ & $74(31)$ & \\
\hline Partner education level & & & & 0.59 \\
\hline Less than college degree & $48(30)$ & $48(29)$ & $66(28)$ & \\
\hline College degree & $66(41)$ & $83(50)$ & $106(44)$ & \\
\hline Some graduate/masters & $28(18)$ & $23(14)$ & $47(20)$ & \\
\hline Completed post-graduate & $17(11)$ & $12(7)$ & $20(8)$ & \\
\hline
\end{tabular}

${ }^{a}$ Fisher's exact test 


\section{Table 2}

Day-specific probability fecundability ratios (FR) with $95 \%$ credible intervals (CI) by number of days with intercourse during the peri-implantation window.

\begin{tabular}{|l|c|c|c|c|}
\hline Number of Days & \multicolumn{2}{|c|}{$\begin{array}{c}\text { Fecundability Ratio } \\
\text { Unadjusted }\end{array}$} & \multicolumn{2}{|c|}{$\begin{array}{c}\text { Fecundability Ratio } \\
\text { Adjusted } \boldsymbol{a}\end{array}$} \\
\hline & FR & $\mathbf{9 5 \%}$ CI & FR & 95\% CI \\
\hline None & \multicolumn{5}{|c|}{ Reference } \\
\hline 1 day & 0.97 & $(0.65-1.39)$ & 0.98 & $(0.66-1.40)$ \\
\hline 2 days & 0.74 & $(0.45-1.12)$ & 0.76 & $(0.47-1.15)$ \\
\hline 3 or more days & 0.48 & $(0.28-0.75)$ & 0.52 & $(0.30-0.82)$ \\
\hline
\end{tabular}

${ }^{a}$ Adjusted for age, BMI, race, presence of regular menstrual cycles, and previous pregnancy 